\title{
Coelomomyces psorophorae Couch, 1945 \\ (Blastocladiales - Coelomomycetaceae), \\ parasite d'Aedes detritus (Haliday, 1833) \\ (Diptera-Culicidae) en Camargue
}

\author{
par E. GUILVARD, J.-A. RIOUX, J.-F. MANIER et J. PECH-PERIERES \\ (Collaboration technique: A. RODRIGUEZ et S. OUSTAU) \\ Laboratoire d'Ecologie médicale et Pathologie parasitaire ( $\mathrm{P}^{r}$ J.-A. Rioux), \\ Faculté de Médecine, F 34000 Montpellier \\ et Laboratoire de Zoologie 1, U.S.T.L., F 34060 Montpellier Cedex
}

\section{Résumée.}

Le Phycomycète parasite, Coelomomyces psorophorae Couch, 1945, est retrouvé dans le sud de la France, chez le Culicide halophile Aedes detritus (Haliday, 1833). Le Champignon occupe les ovarioles en laissant intact le follicule. Examinées à fort grossissement, les ponctuations du sporange apparaissent comme des invaginations cratériformes au fond desquelles se différencie l'ostiole d'un canalicule intéressant la totalité de la paroi externe.

\section{Summary.}

Coelomomyces psorophorae Couch, 1945 (Blastocladiales-Coelomomycetaceae), a parasite of Aedes detritus (Haliday, 1833) (Diptera-Culicidae) in the Camargue.

The Phycomycete, Coelomomyces psorophorae Couch, 1945, was found in the south of France in the salt marsh mosquito Aedes detritus (Haliday, 1833). The fungus invades the ovarioles but leaves the follicules intact. At high magnification, (using a scanning electron microscope), the crater-form punctuations adorning the sporangium were seen to result from openings (ostioles) of the network of small canals that traverse the external wall.

Accepté le 22 septembre 1976. 
Les organismes entomopathogènes, objet principal de nos activités de recherches, sont particulièrement nombreux au sein des populations méditerranéennes de Diptères Culicides. Ainsi, l'espèce halophile Aedes detritus (Haliday, 1833) héberge un virus de type irisant (C. Vago et coll., 1969), plusieurs Microsporidies (S. Tour et coll., 1971), des Trichomycètes (J.-F. Manier et coll., 1964) ainsi qu'un Nématode Mermithidae (M.-M. Doucet et coll., 1976). Récemment, la dissection systématique de femelles (1) récoltées en Camargue nous a permis de dépister un Champignon Phycomycète, parasite des ovaires, Coelomomyces psorophorae Couch, 1945. Malgré la rareté du matériel, une première série d’observations, morphologiques et écologiques, a pu être réalisée.

\section{Matériel et méthodes}

\section{Biotopes larvaires.}

Parmi les gîtes larvo-nymphaux inventoriés sur l'ensemble du delta du Rhône, trois seulement se sont montrés infestés. Nous en donnons la description succincte :

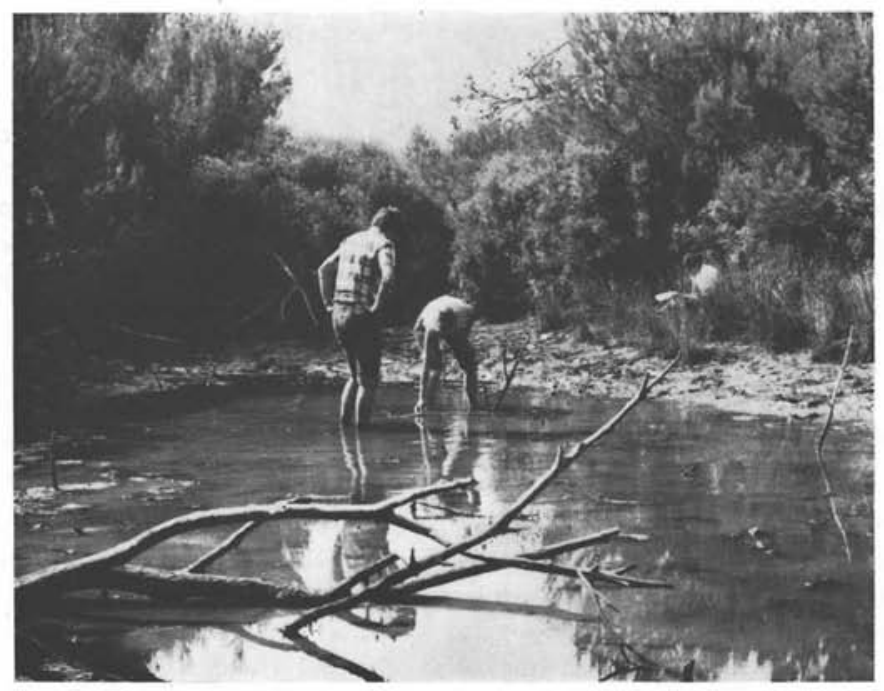

FIG. 1. - Mare temporaire formée dans une dépression interdunaire, biotope larvaire à Aedes detritus (Haliday, 1833) parasitée par Coelomomyces psorophorae Couch, 1945. En bordure, Juncus acutus, Phyllirea angustifolia et Pinus halepensis.

(1) Réalisée dans le cadre d'une étude éco-physiologique de l'autogénèse. 
Gîtes de Petite-Camargue (fig. 1). Les deux gîtes contaminés sont constitués par des dépressions interdunaires de faible profondeur, disséminées dans la forêt mixte de Pin pignon (Pinus pinea), Pin d'Alep (Pinus halepensis) et Genévrier de Phénicie (Juniperus phoenicae). Le sous-bois est composé de Romarin (Rosmarinus officinalis), de Phylaire (Phyllirea angustifolia), de Lentisque (Pistacia lentiscus) et de Ciste à feuilles de Sauge (Cistus salviaefolius). La bordure du gîte est occupée par une végétation semi-halophile représentée essentiellement par Juncus maritimus, Statice limonium et Statice virgatum. Les eaux libres hébergent l'hydrophyte Ruppia maritima. Après enfoncement de la nappe, les annuelles halophiles (Sueda maritima et Salicornia annua) s'emparent des zones exhondées. Au moment du premier prélèvement de nymphes, la teneur en $\mathrm{Cl} \mathrm{Na}$ était de $20,4 \mathrm{~g}$ /litre. La fréquence du parasite a été successivement, au cours du printemps 1976 de $4,41 \%$ le 28 avril, $0,57 \%$ le 13 mai et $5,14 \%$ le 18 mai.

Gîtes de Grande-Camargue. Ce gîte est situé près des tables de préconcentration, dans l'exploitation salinière de Salins-de-Giraud. Il s'agit d'un séquestre provenant du colmatage d'une zone de prélèvement bordant un chemin de terre. En dehors des grandes étendues d'eaux saumâtres, les formations végétales sont représentées par des landes à Chénopodiacées («sansouires») où dominent Salicornia fruticosa, Salicornia radicans et Arthrocnemum glaucum (J.-A. Rioux, 1958). Au moment du prélèvement, le 13 mai 1976, la teneur en $\mathrm{Cl} \mathrm{Na}$ était de $11 \mathrm{~g} /$ litre et la fréquence du parasite de $1,44 \%$.

\section{Conditionnement des imagos femelles.}

Le matériel examiné provient de nymphes prélevées directement sur le terrain. Pour ce faire, chaque nymphe est placée dans un récipient de carton paraffiné. Dès l'éclosion, les imagos femelles sont transférées dans un pot de verre (hauteur: $15 \mathrm{~cm}$, diamètre: $5 \mathrm{~cm}$ ) à fond de plâtre, imbibé d'eau distillée. Elles sont nourries exclusivement d'eau sucrée. Les exemplaires non autogènes sont disséqués au $20^{\circ}$ jour. Les ovaires sont examinés de manière à préciser l'état d'évolution du vitellus (échelle de Christophers).

\section{Etude du matériel parasitaire.}

EXAmen AU microscope photonique. Il se pratique soit directement, dans l'eau chlorurée à $6 \%$ ou après coloration au Bleu Coton (montage au polyvinyllactophénol), soit après fixation au tétroxyde d'osmium, montage dans l'Araldite ${ }^{\circledR}$ (coupe semi-fine) et coloration selon la technique de J.-P. Musy et coll. (1970).

EXAMEN AU MiCROSCOPE ÉlECTRONIQUE. L'observation au microscope à balayage nécessite la dissection des ovaires. Les sporanges, libérés des ovarioles, sont pré- 

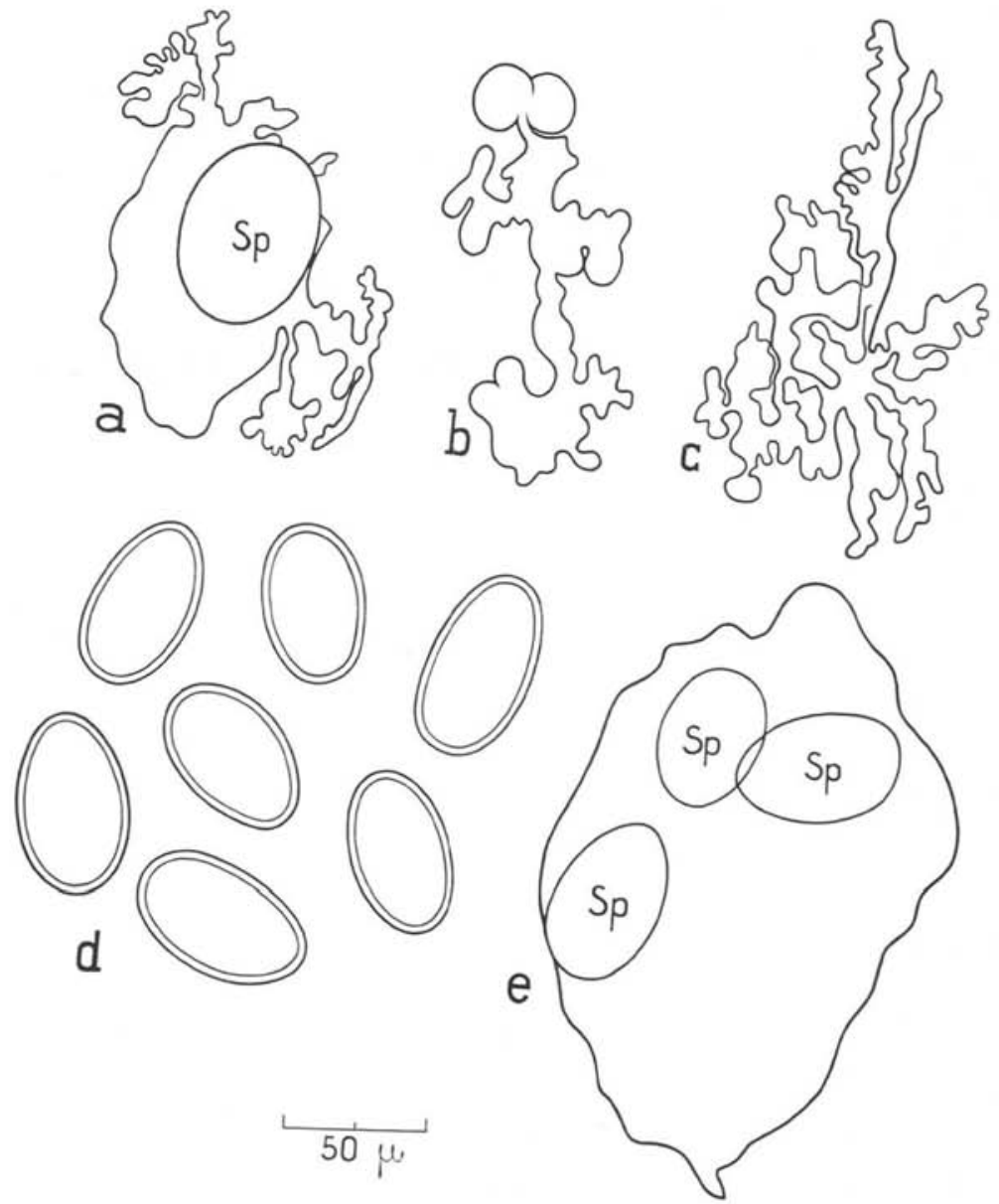

Fig. 2. - Coelomomyces psorophorae Couch, 1945. Rangée supérieure: thalle coralliforme $(b$ et $c)$ et sporange $(a)$. Rangée inférieure : sporanges libres $(d)$ et intraovariens $(e)$.

levés individuellement à la pipette à double effilure. Ils sont ensuite déposés dans une goutte d'eau distillée, lavés à plusieurs reprises, collés sur une lame porte-objet, désséchés pendant une heure puis métallisés. L'examen proprement dit est réalisé au microscope électronique à balayage Jéol, type SM 35. L'observation au microscope à transmission se pratique sur des coupes ultra-fines. Les ovaires fixés au tétroxyde d'osmium $\left(p \mathrm{H} \mathrm{7,4)}\right.$ et déshydratés sont inclus dans l'Araldite ${ }^{\circledR}$. Les coupes ultra-fines, traitées par l'acétate d'uranyle et contrastées au citrate de plomb sont examinées au microscope électronique à transmission Zeiss, type EM 9A. 


\section{Résultats et discussion}

\section{Le Parasite.}

LE THALLE est constitué par un mycólium coenocytique à ramifications coralliformes (fig. $2 \mathrm{a}, \mathrm{b}$ et c), caractéristiques des représentants du genre Coelomomyces. In situ, le mycélium apparaît replié sur lui-même, formant un amas compact périfolliculaire (fig. 8). Les hyphes, de diamètre irrégulier, sont limitées par la seule membrane unitaire, d'aspect plissé (fig. 10). Elles renferment de nombreux noyaux, souvent en division selon le mode crypto-mitotique de A. Hollande (1972). Les mitochondries, très allongées, sont parfois ramifiées. Les globules lipidiques, polymorphes, se distribuent par amas. Des séries de saccules, aplatis et orientés parallèlement, évoquent des formations golgiennes (fig. 3).

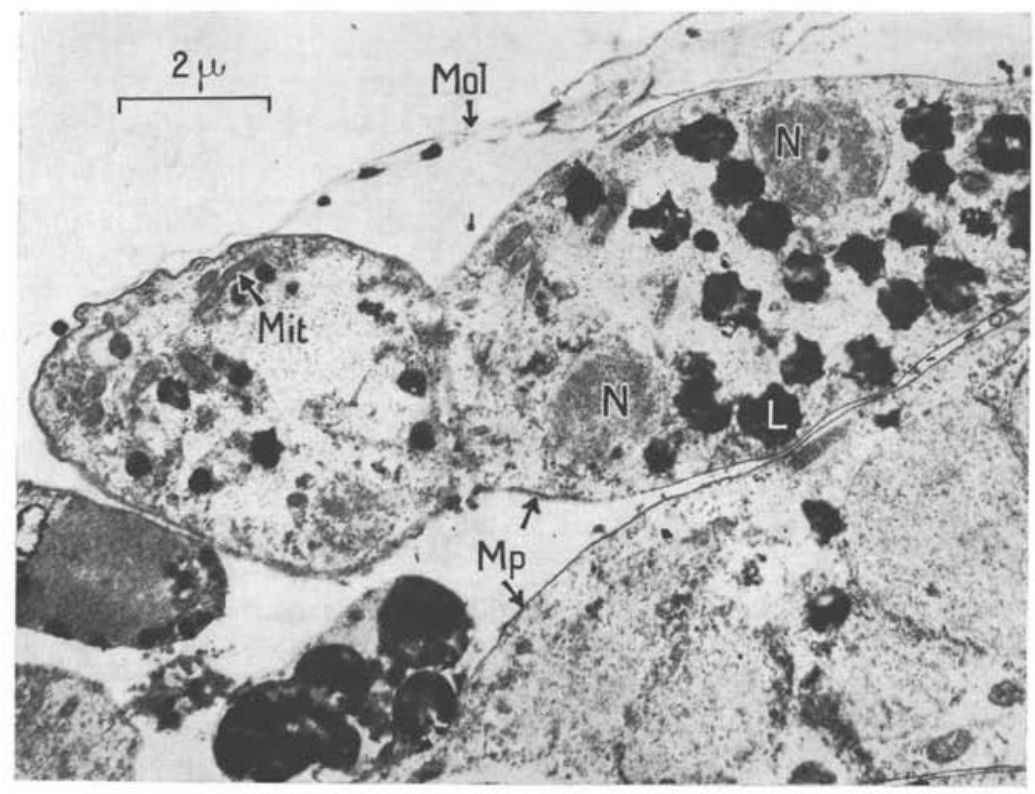

FIG. 3. - Coelomomyces psorophorae Couch, 1945. Portion du thalle montrant l'aspect moniliforme et la structure coenocytique. L: globule lipidique; Mit: mitochondrie; Mol : membrane ovariolaire. Mp : membrane plasmique du thalle. $\mathrm{N}$ : noyau [mic. élec. transm.].

Le SPORANGe (fig. 2 d), ovoïde, de 65 à $110 \mu$ de long sur 40 à $75 \mu$ de large, présente une légère asymétrie axiale. 
Au microscope photonique, la paroi apparaît constituée de deux enveloppes, l'une externe, épaisse de 2 à $3 \mu$, régulièrement ponctuée, l'autre interne, mince et transparente (fig. $4 \mathrm{a}$ et $\mathrm{b}$ ). Le bouchon de déhiscence, subpolaire, est constitué d'une substance amorphe paraissant dépendre de l'enveloppe interne. A l'intérieur du sporange, se différencient les zoospores.

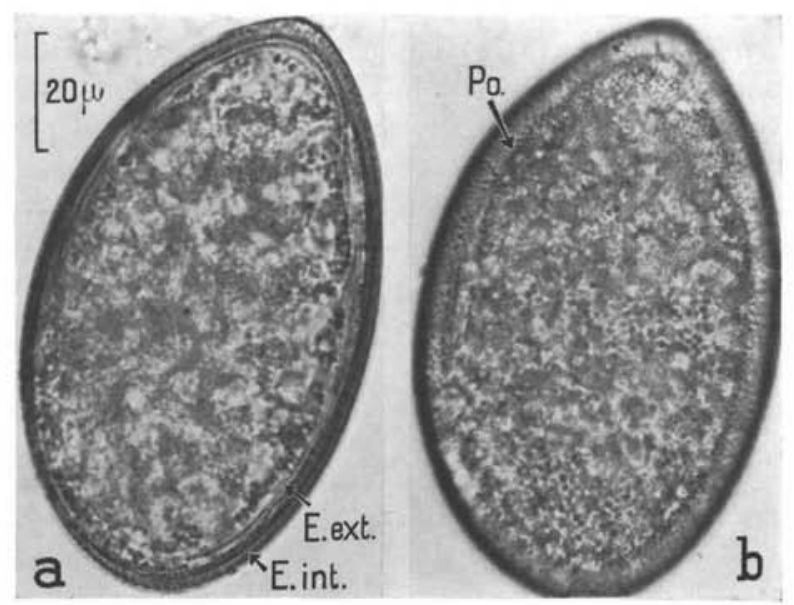

Fig. 4. - Coelomomyces psorophorae Couch, 1945. sporange: a) en coupe frontale, traversant les enveloppes externe (E. ext.) et interne (E. int.); $b$ ) en vue supérieure, montrant les ponctuations caractéristiques.

Au microscope électronique à balayage, les ponctuations se présentent comme des microdépressions cratériformes, donnant un aspect général « en peau d'orange » (fig. 6 a). En leur centre, se différencie l'orifice externe d'un canalicule (fig. $6 \mathrm{c}$ ). Entre les dépressions, la surface du sporange présente un aspect gaufré (fig. 6 b et c). Une strie longitudinale, interprétée par J.N. Couch (1945) comme une fente de déhiscence, est parfois visible (fig. 5).

Au microscope électronique à transmission, la paroi apparaît constituée de deux strates (fig. 7 a), l'une interne, mince et peu osmiophile (secondary wall de H. C. Whisler et coll., 1972), l'autre, externe, épaisse et structurée (primary wall de H. C. Whisler et coll., 1972). De l'extérieur vers l'intérieur, cette dernière se différencie en quatre couches: une couche mince et continue, une couche épaisse parcourue de travées transparentes et s'ouvrant à l'extérieur par les orifices en pavillons décrits ci-dessus (fig. 7 a et b), une couche interne d'aspect alvéolaire (fig. 7 c), limitée en profondeur par une couche dense et continue.

\section{Relations Hôte-Parasite.}

Sur le frais, les ovaires parasités apparaissent hypertrophiés et d'aspect lactescent. Par transparence, on distingue des formations ovalaires, de couleur jaune ocre: les 


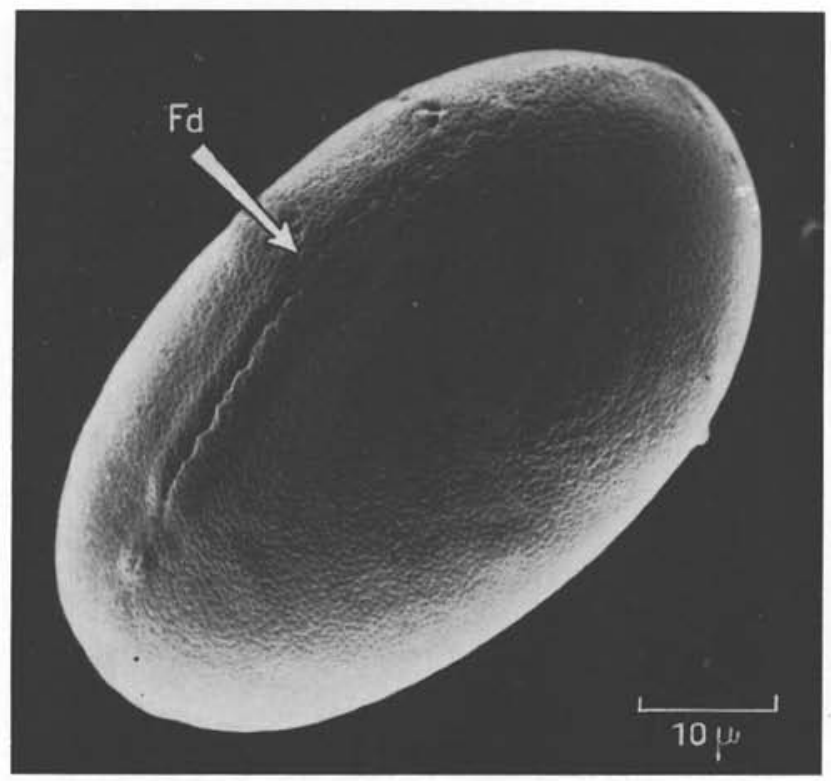

FIG. 5. - Coelomomyces psorophorae Couch, 1945. Sporange montrant l'ornementation de l'enveloppe et la fente de déhiscence (Fd) [mic. élec. bal.].

sporanges. Après dilacération, l'ovariole parasitée se présente sous la forme d'un sac aux parois distendues (fig. 8). Les follicules, enveloppés par le mycélium, sont difficilement visibles. La rupture de la membrane ovariolaire permet au thalle de s'étaler, cependant que les sporanges se détachent. Les follicules ovariens, arrêtés au stade II, apparaissent alors indemnes de parasites. Cette situation extra-folliculaire est confirmée par l'examen des coupes.

Effectivement, sur les coupes semi-fines (microscope photonique) et ultra-fines (microscope électronique à transmission), le parasite apparaît localisé à l'intérieur des ovarioles et en dehors des follicules (fig. 8). Il occupe la cavité virtuelle de la séreuse limitée par la tunica propria et la membrane ovariolaire (fig. 9). Aucun sporange n'est présent dans les oviductes ainsi qu'il est mentionné dans l'observation de P. T. Lum (1963). Les follicules parasités sont totalement enveloppés par le thalle, mais on ne note aucun phénomène de lyse ou de nécrose, tant au niveau des cellules folliculaires que de l'oocyte (fig. 11). Sur nos exemplaires, ce dernier ne dépasse jamais le stade II de la vitellogénèse. A ce propos, on peut se demander s'il s'agit d'un blocage physiologique, normal chez les femelles anautogènes, et par conséquent si l'évolution ultérieure jusqu'au stade $\mathrm{V}$ aurait pu se poursuivre après repas de sang. Selon P. T. Lum (loc. cit.), des femelles d'Aedes taeniorhynchus (Wiedemann, 1821), 

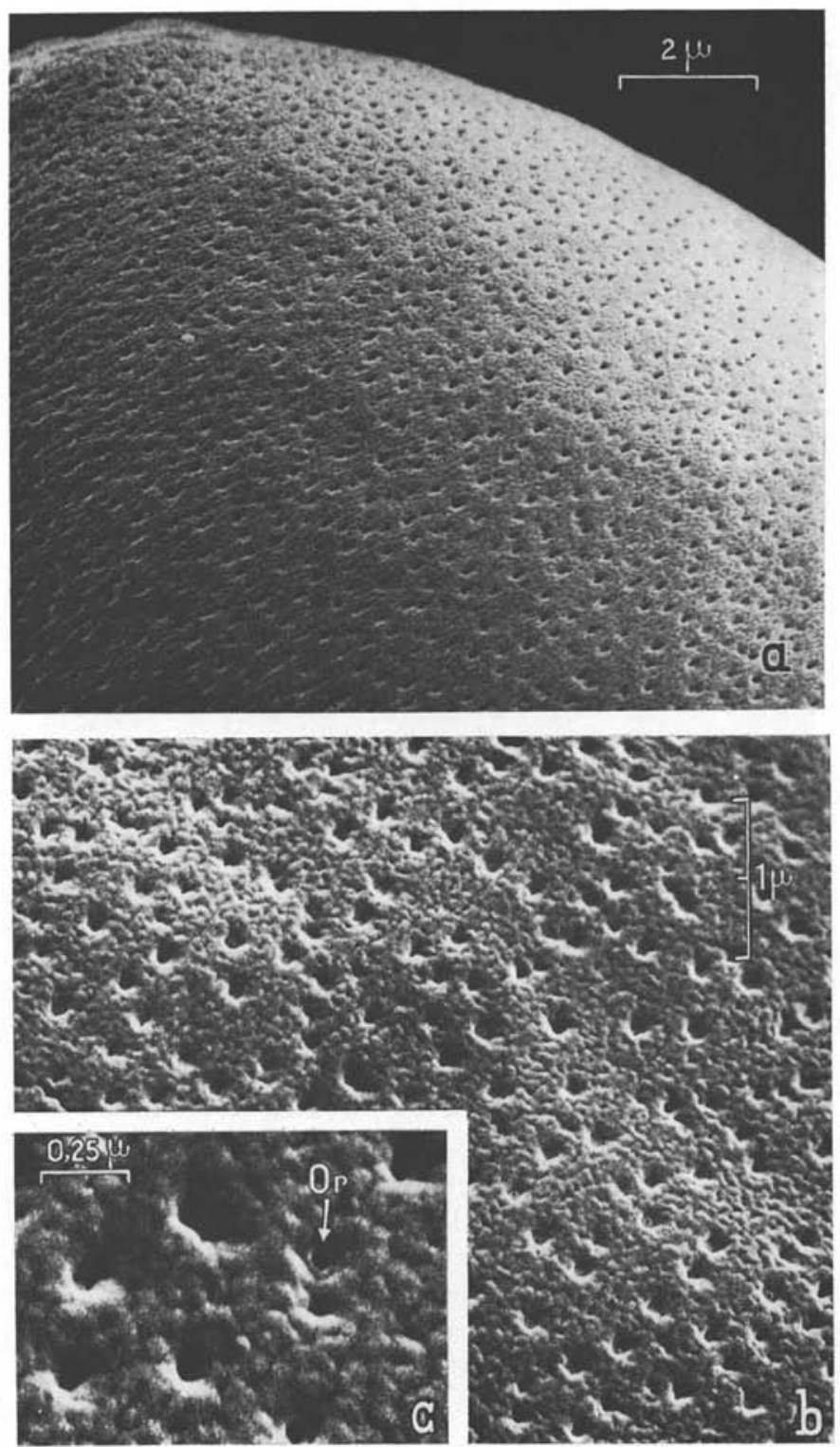

Fig. 6. - Coelomomyces psorophorae Couch, 1945. Surface du sporange montrant les ponctuations « en peau d'orange 》 $(a)$. Vues à un plus fort grossissement, celles-ci apparaissent sous la forme de dépressions cratériformes ( $b$ et $c$ ), au fond desquelles se distingue l'orifice d'un canalicule transpariétal (Or) [mic. élec. bal.]. 

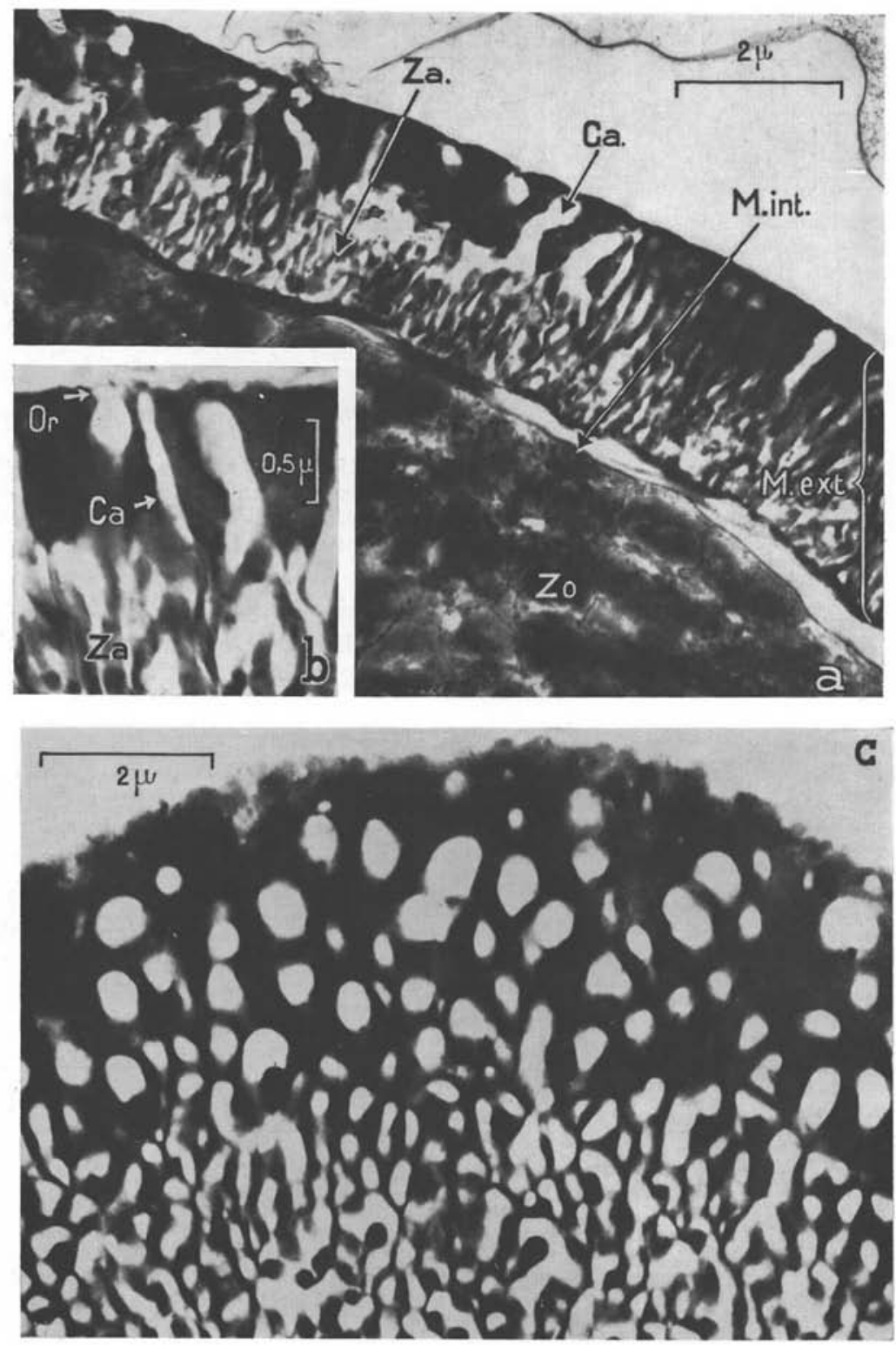

FIg. 7. - Coelomomyces psorophorae Couch, 1945. Structure de la paroi sporangiale. L'enveloppe externe $(a)$ montre une couche superficielle dense, traversée de canalicules s'ouvrant à l'extérieur par des orifices campanulés $(b)$ et se prolongeant vers l'intérieur dans une formation labyrinthique d'aspect alvéolé. La structure de cette zone est particulièrement nette sur les coupes tangentielles $(c)$. Par ailleurs les ébauches de zoospores sont visibles au-dessous de la membrane interne. Ca: canalicule; M. ext. : membrane externe ; M. int : membrane interne; Or: orifices des canalicules; Za : zone alvéolaire; Zo: zoospore [mic. élec. transm.]. 


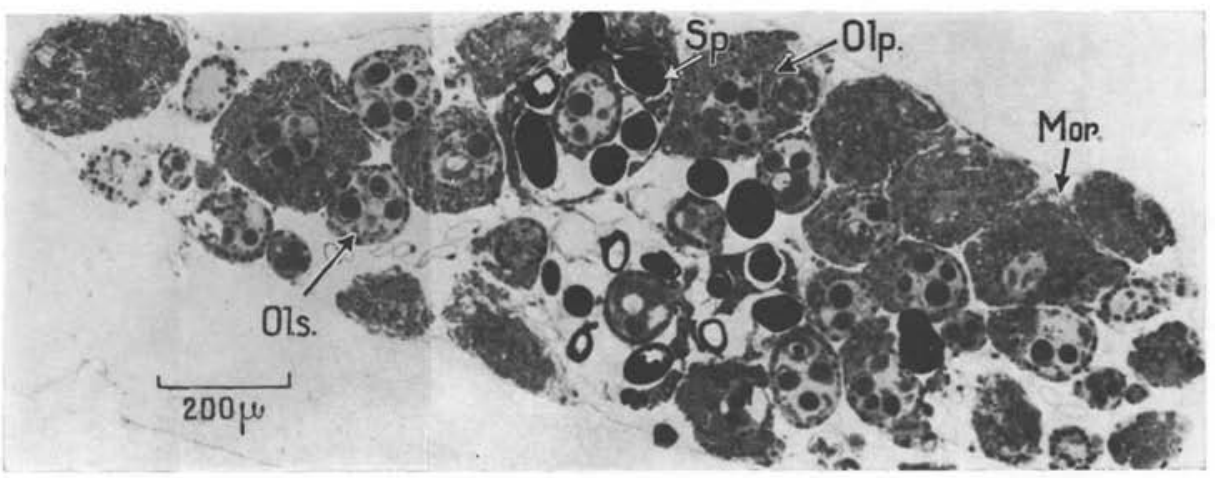

FIG. 8. - Coelomomyces psorophorae Couch, 1945, parasite d'Aedes detritus (Haliday, 1833). Coupe de l'ovaire montrant la disposition intra-ovariolaire du parasite sous la forme de mycelium et de sporanges. Mor: membrane ovarienne; Olp : ovariole parasité ; Ols : ovariole sain; Sp: sporange [mic. élec. transm.].

parasitées, peuvent en effet poursuivre la vitellogénèse jusqu'au stade IV, sans toutefois atteindre la maturité. W. R. Kellen et coll. (1963) identifient, cependant un individu pare sur un lot de sept Aedes melanimon Dyar, 1924 infestées.

\section{Spectre d'hôtes.}

Décrit en 1945 par J. N. Couch chez les larves de Psorophora ciliata (Fabricius, 1794), Coelomomyces psorophorae a été retrouvé chez Psorophora howardii Coquilett, 1901, mais également chez d'autres genres : Culiseta inornata (Williston, 1893), Aedes taeniorhynchus (Wiedemann, 1821), Aedes melanimon Dyar, 1924, Aedes vexans (Meigen, 1830) et peut-être Aedes cinereus Meigen, 1818, Aedes scatophagoides (Theobald, 1901) et Culiseta morsitans (Theobald, 1901) (tableau I).

TABleau I. - Répartition géographique et spectre d'hôtes de Coelomomyces psorophorae Couch, 1945.

\begin{tabular}{|c|c|c|c|}
\hline Auteurs & Hôtes & $\begin{array}{c}\text { Stades } \\
\text { parasitées }\end{array}$ & $\begin{array}{l}\text { Localisations } \\
\text { géographiques }\end{array}$ \\
\hline $\begin{array}{l}\text { J. N. Couch, } 1945 \text { (description } \\
\text { princeps). } \\
\text { J. N. Couch et H. R. Dodge, } 1947 \\
\text { J. A. Shemanchuck, } 1959 . \\
\text { M. Laird, 1961. } \\
\text { P. T. Lum, } 1963 . \\
\text { W. R. Kellen et coll., } 1963 . \\
\text { J. Weiser et J. Vavra, } 1964 . \\
\text { A. M. Golberg et coll., } 1975 . \\
\text { ?. E. Ekstein, } 1922 \text {. } \\
\text { ?. Muspratt, } 1946 . \\
\text { ?. A.-R. Barr, 1958. }\end{array}$ & $\begin{array}{l}\text { Psorophora ciliata. } \\
\text { Aedes vexans. } \\
\text { Psorophora howardii. } \\
\text { Culiseta inornata. } \\
\text { Culiseta inornata. } \\
\text { Psorophora ciliata. } \\
\text { Aedes vexans. } \\
\text { Psorophora howardii. } \\
\text { Aedes taeniorhynchus. } \\
\text { Aedes melanimon. } \\
\text { Aedes vexans. } \\
\text { Aedes vexans. } \\
\text { Aedes cinereus. } \\
\text { Aedes vexans. } \\
\text { Aedes scatophagoides. } \\
\text { Culiseta morsitans. }\end{array}$ & $\begin{array}{l}\text { larves. } \\
\text { larves. } \\
\text { larves. } \\
\text { larves. } \\
\text { larves. } \\
\text { larves. } \\
\text { larves. } \\
\text { larves. } \\
\text { adultes } \\
\text { adultes. } \\
\text { larves. } \\
\text { larves. } \\
\text { larves. } \\
\text { larves. } \\
\text { larves. } \\
\text { larves. }\end{array}$ & $\begin{array}{l}\text { U.S.A. (Géorgie). } \\
\text { U.S.A. (Géorgie). } \\
\text { U.S.A. (Caroline du Sud). } \\
\text { U.S.A. (Louisiane). } \\
\text { Canada (Alberta). } \\
\text { U.S.A. (Mississipi). } \\
\text { U.S.A. (Minesota). } \\
\text { U.S.A. (Floride). } \\
\text { U.S.A. (Floride). } \\
\text { U.S.A. (Californie). } \\
\text { Tchécoslovaquie. } \\
\text { U.R.S.S. (A.S.S.R.). } \\
\text { France. } \\
\text { France. } \\
\text { Zambie. } \\
\text { U.S.A. (Minesota). }\end{array}$ \\
\hline
\end{tabular}




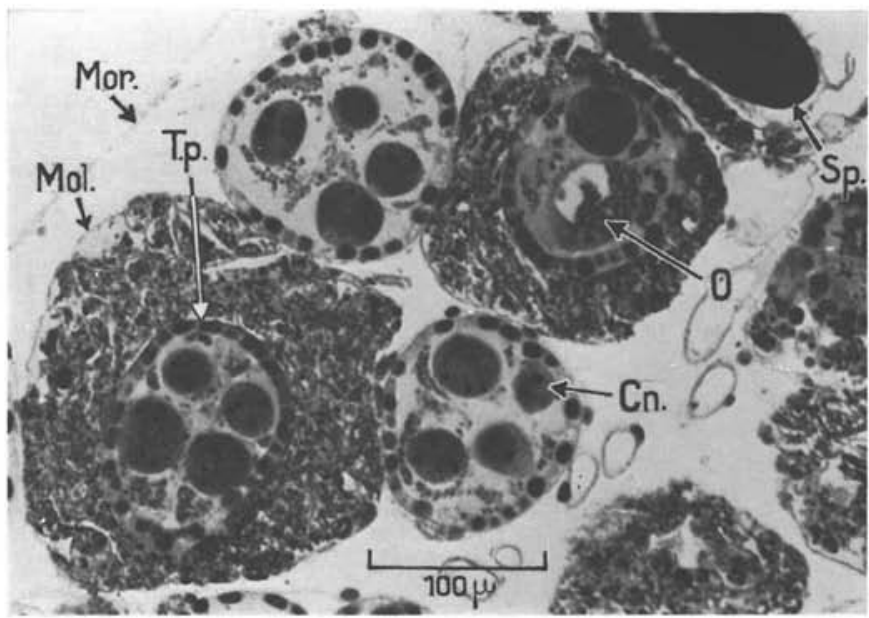

FIG. 9. - Coelomomyces sporophorae Couch, 1945, parasite d'Aedes detritus (Haliday, 1833). Détail de la figure 8 présentant la situation du mycelium entre la membrane ovariolaire et la membrane folliculaire. $\mathrm{Cn}$ : cellule nourricière; Mol : membrane ovariolaire ; Mor: membrane ovarienne; $\mathrm{O}$ : oocyte; $\mathrm{Sp}$ : sporange [mic. élec. transm.].

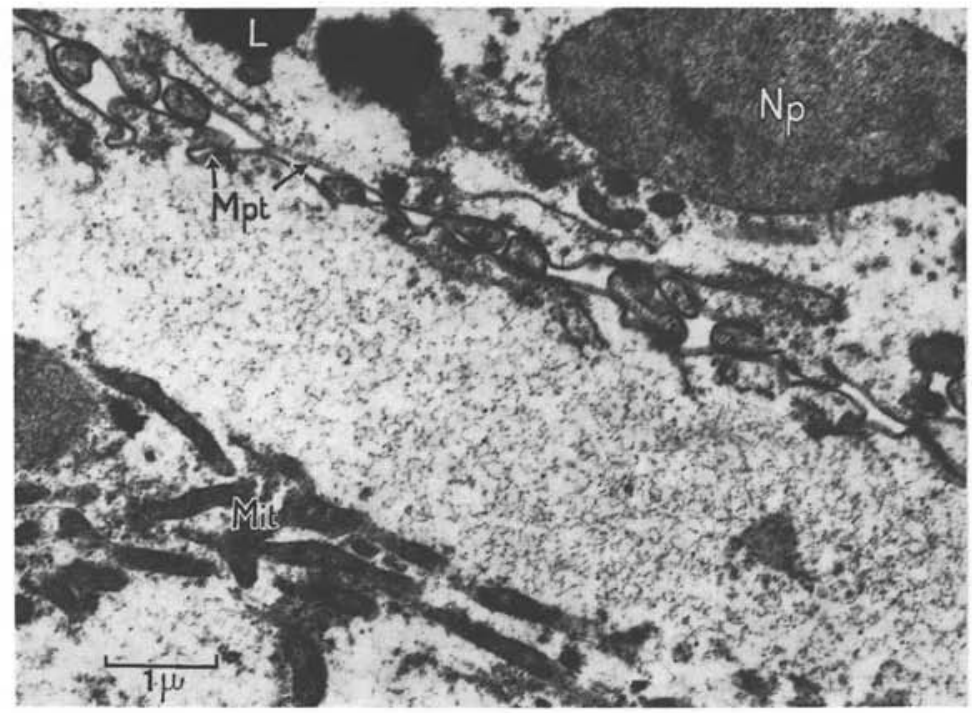

FiG. 10. - Coelomomyces sporophorae Couch, 1945, parasite d'Aedes detritus (Haliday, 1833). Coupe de deux hyphes juxtaposées montrant la membrane plasmique fortement plissée. L : globule lipidique ; Mit : mitochondrie; Mpt : membrane plasmique; Np : noyau du parasite [mic. élec. transm.]. 
Au demeurant, si l'on excepte les observations de P. T. Lum (loc. cit.) et de W. R. Kellen (loc. cit.), Coelomomyces psorophorae n'a été observé que chez les larves (tableau I). Dans ce cas, le parasite occupe la cavité cœlomique, depuis la région céphalique jusqu'au segment anal. En fait, il semble qu'il y ait balancement entre les deux processus. Ainsi, une recherche concomitante de l'infestation larvaire a permis à P.-T. Lum (loc. cit.) de dépister 300 larves parasitées sur un lot de 5000 . Aucune des larves atteintes n'est parvenue à l'âge adulte. L'auteur estime que le parasitisme imaginal n'est pas compatible avec une infestation larvaire précoce. De son côté, W. R. Kellen (loc. cit.) examine, sans succès, plusieurs milliers de larves d'Aedes melanimon récoltées dans une zone d'infestation imaginale. Aucun adulte issu de ces larves n'est infesté.

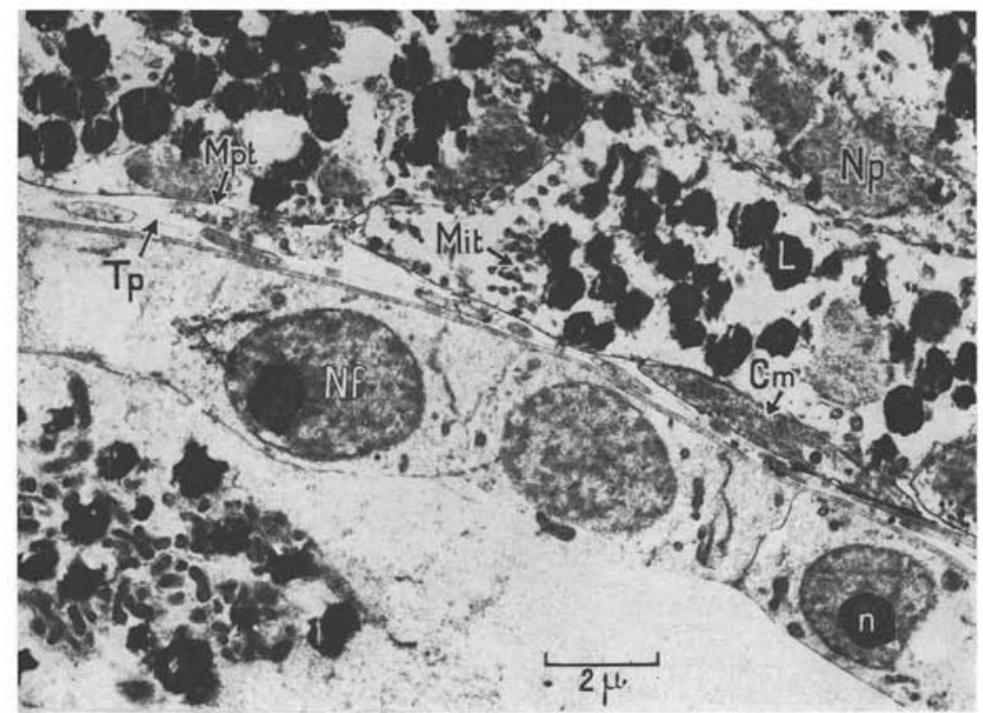

Fig. 11. - Coelomomyces sporophorae Couch, 1945, parasite d'Aedes detritus (Haliday, 1833). Contact follicule-mycelium. Une hyphe (partie supérieure de la figure) est plaquée contre les cellules folliculaires par l'intermédiaire de la tunica propria; $\mathrm{Cm}$ : cellule musculaire périovariolaire; L : globule lipidique ; Mit : mitochondrie ; Mpt : membrane plasmique du thalle; $\mathrm{n}$ : nucléole de la cellule folliculaire; $\mathrm{Np}$ : noyau du parasite ; $\mathrm{Tp}$ : tunica propria [mic. élec. transm.].

Les mentions d'infestations imaginales par Coelomomyces sont effectivement rares. Les toutes premières observations sont rapportées par C. Manalang (Philippines, 1930) chez plusieurs «espèces » d'Anophèles et par L. C. Feng (Chine, 1933) chez Anopheles sinensis Wiedemann, 1828 et Culex tritaeniorhynchus Giles, 1901. Le parasite était situé dans l'hémocèle. E. G. Gibbins (1932), en Uganda, est le premier 
à décrire l'infestation ovarienne chez Anopheles gambiae Giles, 1902 et Anopheles funestus Giles, 1900. A. J. Walker (1938), en Sierra Leone, retrouve une localisation identique chez ces mêmes espèces. Au Kenya, A. J. Haddow (1942) décrit de façon précise une infestation ovarienne par Coelomomyces africanus Walker, 1938, toujours chez Anopheles gambiae et Anopheles funestus. En Indonésie, P. H. Van Thiel (1954) mentionne une infestation par Coelomomyces walkeri Van Thiel, 1954, chez deux femelles d'Anopheles tesselatus Theobald, 1901. A Singapour, M. Laird (1959) observe Coelomomyces quadrangulatus Couch, 1945 dans la cavité générale et les ovaires de Culex tritaeniorhynchus siamensis Barraud et Christophers, 1931. En 1974, F. Rodhain et J. Brengues découvrent, en Haute-Volta, une infestation par Coelomomyces grassei Rioux et Pech, 1960 dans les ovaires d'Anopheles gambiae.

\section{Position systématique.}

Le parasite observé chez Aedes detritus correspond de manière assez précise à la description princeps de J. N. Couch à l'exception toutefois de l'absence d'anastomose entre les perforations de la paroi sporangiale. Il semble cependant qu'une certaine variabilité se rencontre chez cette espèce. En effet, le nombre et la répartition des ponctuations de la paroi sporangiale varient suivant l'hôte d'origine (D. W. Anthony et coll., 1971). Ainsi, pour C. E. Bland et J. N. Couch (1973), Coelomomyces psorophorae constituerait un complexe formé de sous-espèces, voire d'espèces authentiques.

Quoi qu'il en soit, Coelomomyces psorophorae se distingue nettement de Coelomomyces punctatus Couch et Dodge, 1947, dont les perforations sporangiales sont moins nombreuses, plus larges et plus distantes. De plus, au microscope à balayage (D. W. Anthony et coll., loc. cit.), la surface apparaît finement côtelée. L'examen de la paroi sporangiale, selon cette technique, semble d'ailleurs constituer la méthode diagnostique la plus fidèle. Ainsi, l'étude de 18 espèces de Coelomomyces a permis à C. E. Bland et J. N. Couch (1973) de distinguer 8 groupes. Coelomomyces punctatus rangé, au côté de Coelomomyces dodgei Couch et Dodge, 1947 et Coelomomyces lativittatus Couch et Dodge, 1947, dans le groupe $\mathrm{n}^{\circ} 2$, est caractérisé par une paroi côtelée et irrégulièrement ponctuée. Cet aspect avait d'ailleurs été observé en microscopie à transmission par W. W. Martin (1969). Par contre, la différenciation entre, d'une part Coelomomyces psorophorae, d'autre part Coelomomyces stegomyiae Keilin, 1921, Coelomomyces keilini Couch et Dodge, 1947, Coelomomyces tasmaniensis Laird, 1956 et Coelomomyces opifexi Pillai et Smith, 1968 réunis dans le groupe $\mathrm{n}^{\circ} 3$ par C. E. Bland et J. N. Couch (loc. cit.), paraît beaucoup plus subtile. Coelomomyces stegomyiae montre des sporanges ovalaires asymétriques (une face plane, une face convexe) dont la paroi est ornée de ponctuations peu nombreuses (6 à 15 aux $10 \mu^{2}$ ) en forme de dépressions évasées, perforées en leur centre, ainsi que de «granulations ovalaires » irrégulièrement dispersées. Coelomomyces keilini, décrit à partir d'une seule larve d'Anopheles crucians, Wiedemann, 1828, possède également des sporanges. ovales et une face légèrement aplatie; toutefois, la surface est ornée de pertuis stellaires. Coelomomyces tasmaniensis paraît lui aussi très proche de Coelomomyces 
psorophorae. Le sporange à paroi finement ponctuée est également asymétrique. Il est de même difficile de distinguer Coelomomyces opifexi de Coelomomyces psorophorae malgré les tentatives de J. S. Pillai et J. M. Smith (1968).

Autre critère: la taille du sporange ne paraît pas être un élément d'une réelle valeur systématique. On a voulu séparer les espèces à grands sporanges (supérieurs à $60 \times 90 \mu$ ), telles que Coelomomyces psorophorae et Coelomomyces tasmaniensis, des espèces à petits sporanges (inférieurs à $60 \times 90 \mu$ ), telles que Coelomomyces stegomyiae et Coelomomyces keilini. En fait, chez Coelomomyces psorophorae, les dimensions varient de façon notable. Selon J. N. Couch et H. R. Dodge (1947), les sporanges développés chez Aedes vexans sont plus petits $(43-66 \mu \times 63-94 \mu)$, avec une paroi plus mince $(5,5 \mu)$. A l'inverse, ces mêmes auteurs mentionnent chez Culiseta inornata des sporanges de grande taille. De même, D. W. Anthony et coll. (loc. cit.) observent d'importantes variations de taille sur des lots de Coelomomyces psorophorae provenant de cinq hôtes différents. En définitive, il paraît probable que cette espèce regroupe des entités taxonomiques distinctes dépendant des hôtes et plus encore des localités géographiques. Remarquons à ce titre que Coelomomyces psorophorae s'étend de l'Amérique du Nord (U.S.A., Canada) à l'Europe (France, Tchécoslovaquie, U.R.S.S.) et peut-être à l'Afrique. Rappelons que F. Eckstein (1922) aurait observé le parasite en France chez Aedes cinereus et Aedes vexans; J. Muspratt (1946) l'aurait dépisté en Zambie chez Aedes scatophagoides.

\section{Conclusion}

Décrits depuis plus de cinquante ans, les Coelomomyces continuent à poser de passionnants problèmes de systématique, d'écologie et d'épidémiologie. Considérés comme très prometteurs en lutte biologique, ils n'ont pu être utilisés en vraie grandeur en raison de l'échec des cultures de masse et ce, malgré les tentatives de J. Muspratt (1963), de M. F. Madelin (1965), de M. Laird (1967) et de J. N. Couch (1972). En fait, contrairement aux affirmations de nombreux auteurs, il est à présent démontré que la zoospore n'est pas l'unité infestante pour le Moustique. Utilisant le couple Coelomomyces psorophorae-Aedes taeniorhynchus, B. A. Federici et D.W. Roberts (1975) avaient suspecté l'existence d'un hôte intermédiaire, hypothèse avalisée par H. C. Whisler et coll. (1974) qui démontrent l'implication du Crustacé Cyclops vernalis: les zoospores issues de la germination des sporanges effectuent un deuxième cycle obligatoire chez le Copépode. Une nouvelle confirmation des travaux précédents vient d'être apportée par B. A. Federici et D. W. Roberts (1976), à l'aide de Coelomomyces punctatus et Anopheles quadrimaculatus Say, 1924. C'est dire l'intérêt pratique que peut avoir la découverte de Coelomomyces psorophorae dans le * midi » méditerranéen, particulièrement sur les côtes du Languedoc-Roussillon, zone traditionnelle de lutte anticulicidienne. 


\section{Bibliographie}

Anthony (D. W.), Chapman (H. C.) et Hazard (E. I.), 1971. - Scanning electron microscopy of the sporangia of species of Coelomomyces (Blastocladiales: Coelomomycetaceae). J. Invertebr. Pathol., 17, 395-403.

BARR (A. R.), 1958. - The mosquitoes of Minnesota (Diptera ; Culicidae; Culicinae). Tech. Bull., 228, 154 p.

BLAND (C. E.), et Couch (J. N.), 1973. - Scanning electron microscopy of sporangia of Coelomomyces. Can. J. Bot., 51, 1325-1330.

Couch (J. N.), 1945. - Revision of the genus Coelomomyces parasitic in insect larvae. J. Elisha Mitchell Scient. Soc., 61, 124-136.

Couch (J. N.), 1972. - Mass production of Coelomomyces a fungus that kills mosquitoes. Proc. Nat. Acad. Sci., U.S.A., 69, 2043-2047.

Couch (J. N.) et Dodge (H. R.), 1947. - Further observations on Coelomomyces parasitic mosquito larvae. J. Elisha Mitchell Scient. Soc., 63, 69-79.

Couch (J. N.) et Umphlett (C. J.), 1963. - Coelomomyces inrections. J. Insect Pathol., 2, 150-187.

Doucet (M.-M.), Lhaumond (C.), Bain (O.), Rioux (J.-A.) et Guilvard (E.), 1976. — Infestation par Isomermis d'Aedes detritus (Haliday) de Camargue. Com. Soc. fr. Parasit., Strasbourg, 25 mai 1976.

EcksteIn (F.), 1922. - Beitrage zur Kenntnis der Stechmuckenparasiten. Zentbl. Bakt. Abt. Orig., $88,128-135$.

FEDERICI (B. A.) et RoBerTs (D. W.), 1975. - Experimental laboratory infection of mosquito larvae with fungi of the genus Coelomomyces. I. Experiments with Coelomomyces psorophorae var. in Aedes taeniorhynchus and Coelomomyces psorophorae var. in Culiseta inornata. J. Invertebr. Pathol., 26, 21-27.

FEDERICI (B. A.) et RoberTs (D. W.), 1976. - Experimental laboratory infection of mosquito larvae with fungi of the genus Coelomomyces. II. Experiments with Coelomomyces punctatus in Anopheles quadrimaculatus. J. Invertebr. Pathol., 27, 21-27.

FENG (L. C.), 1933. - Some parasites of mosquitoes and flies found in China. Lingan Sci. J., 47, 1191-1199.

GibBins (E. G.), 1932. - Natural malaria infection of house-frequenting Anopheles mosquitoes in Uganda. Ann. trop. Med. Parasit., 26, 239-266.

Golberg (A. M.), Markovich (N. Y.) et Praskuryakova (A. M.), 1975. - A case of detection of Coelomomyces psorophorae Couch (Phycomycetes, Blastocladiales) a parasite of Aedes vexans in Tuva A.S.S.R., Medskaya Parasit., 44, 103-104.

Haddow (A. J.), 1942. - The mosquito fauna and climate of native huts at Kisimu Kenya. Bull. ent. Res., 33, 91-142.

Hollande (A.), 1972. - Le déroulement de la cryptomitose et les modalités de la ségrégation des chromatides dans quelques groupes de Protozoaires. Ann. Biol., 11, 428-466.

Kellen (W. R.), Clark (T. B.) et Lindegren (J. E.), 1963. - A new host record for Coelomomyces psorophorae Couch in California (Blastocladiales: Coelomomycetaceae). J. Insect Pathol., 5, 167-173. 
LAIRD (M.), 1956. - A new species of Coelomomyces (Fungi) from tasmanian mosquito larvae. J. Parasitol., 42, 53-55.

LAIRD (M.), 1959. - Parasites of Singapore mosquitoes with particular references to the significance of larval epibionts as an index of habitat pollution. Ecology, 40, 206-220.

LAIRD (M.), 1961. - New american locality records for four species of Coelomomyces (Blastocladiales, Coelomomycetaceae). J. Insect Pathol., 3, 249-253.

LAIRD (M.), 1967. - Expériences sur des atolls. De nouvelles méthodes de lutte contre les moustiques. Chronicle Hlth Org., 21, 19-28.

LUM (P. T.), 1963. - The infection of Aedes taeniorhynchus (Wiedemann) and Psorophora howardii Coquillett by the fungus Coelomomyces. J. Insect Pathol., 5, 157-166.

MAdELIN (M.F.), 1965. - Further laboratory studies on a species of Coelomomyces which infects Anopheles gambiae. Giles. WHO/Mal/5.

Manalang (C.), 1930. - Coccidiosis in Anopheles mosquitoes. Philipp. J. Sci., 42, 279-282.

Manier (J.-F.), Rioux (J.-A.) et Juminer (B.), 1964. - Présence en Tunisie de deux Trichomycètes parasites de larves de Culicides. Arch. Inst. Pasteur, Tunis, 41, 147-152.

MarTin (W. W.), 1969. - A morphological and cytological study of the development of Coelomomyces punctatus parasitic in Anopheles quadrimaculatus. J. Elisha Mitchell Sci. Soc., 85, 59-72.

Muspratt (J.), 1946. - On Coelomomyces fungi causing hight mortality of Anopheles gambiae larvae in Rhodesia. Ann. trop. Med. Parasit., 40, 10-17.

MuspratT (J.), 1963. - Rapport sur l'état d'avancement (en mai 1963) des enquêtes sur trois agents pathogènes de Moustiques à Livingstone, Rodhésie du Nord, WHO/ vector control/45.

Musy (J.-P.), Modis (L.), Gotzos (V.) et Gondi (G.), 1970. - Nouvelles méthodes de coloration sur coupes semi-fines pour tissus inclus en Araldite. Etude au microscope à champ clair, à contraste de phase et à fluorescence. Acta Anat., 77, 37-49.

Nolan (R. A.), 1973. - Effects of plant hormones on germination of Coelomomyces psorophorae resistant sporangia. J. Invertebr. Pathol., 21, 26-30.

Pillai (J. S.) et SMith (J. M.), 1968. - Fungal pathogens of mosquitoes in New Zealand. I. Coelomomyces opifexi sp. n. on the mosquito, Opifex fuscus Hutton. J. Invertebr. Pathol., 11, 316-320.

Rıoux (J.-A.), 1958. - Les Culicides du \& Midi » méditerranéen. Etude systématique et écologique. Lechevallier édit., Paris, 303 p.

Roberts (D. W.), Shapiro (M.) et Rodin (R. L.), 1973. - Dehiscence of Coelomomyces psorophorae sporangia from Aedes taeniorhynchus: induction by amines and amino acids. J. Inverterbr. Pathol., 22, 175-181.

RodHain (F.), 1968. - Eléments d'une révision des champignons du genre Coelomomyces, parasites des moustiques. Thèse Fac. Méd. Paris, 87 p. (dactylo).

Rodhain (F.) et Brengues (J.), 1974. - Présence de champignons du genre Coelomomyces chez des Anophèles en Haute-Volta. Ann. Parasitol. hum. comp., 49, 241-246.

SchemanchuK (J. A.), 1959. - Note on Coelomomyces psorophorae Couch, a fungus parasitic on mosquito larvae. Can. Ent., 91, 743-744.

SHAPIRO (M.) et ROBERTS (D. W.), 1976. - Growth of Coelomomyces psorophorae mycelium in vitro. J. Invertebr. Pathol., 27, 399-402. 
Tour (S.), Rioux (J.-A.) et Croset (H.), 1971. - Systématique et écologie des Microsporidies (Microsporidia-Nosematidae), parasites de larves de Culicides (Diptera-Culicidae). Enquête sur les espèces du «Midi» méditerranéen. Stempellia tuzetae n. sp. Ann. Parasitol. hum. comp., 46, 205-223.

VAGo (C.), Rioux (J.-A.) Duthoit (J.-L.) et Dedet (J.-P.), 1969. - Infestation spontanée à virus irisant dans une population d'Aedes detritus (Hal., 1833). Ann. Parasitol. hum. comp., 44, 667-676.

VAN Thiel (P. H.), 1954. - Trematode, Gregarine and fungus parasites of Anopheles mosquitoes. J. Parasitol., 40, 271-279.

WALKer (A. J.), 1938. - Fungal infections of mosquitoes especially of Anopheles costalis. Ann. trop. Med. Parasit., 32, 231-244.

Weiser (J.) et VAVRA (J.), 1964. - Zur Verbreitung der Coelomomyces. Pilze in europaischen Insekten. Z. Tropenmed. Parasit., 15, 38-42.

Whisler (H. C.), Shemanchuck (J. A.) et Travland (L. B.), 1972. - Germination of the resistant sporangia of Coelomomyces psorophorae. J. Invertebr. Pathol., 19, 139-147.

Whisler (H. C.), Zebold (S. L.) et Shemanchuck (J. A.), 1974. - Alternate host for mosquito parasite Coelomomyces. Nature, 251, 715-716.

Whisler (H. C.), Zebold (S. L.) et Shemanchuk (J. A.), 1975. - Life history of Coelomomyces psorophorae. Proc. natr. Acad. Sci. U.S.A., 72, 693-696. 